\title{
CONCEPTS
}

\section{A New Proposal for Management of Severe Frostbite in the Austere Environment}

\author{
Emmanuel Cauchy, MD; Christopher B. Davis, MD; Mathieu Pasquier, MD; Eric F. Meyer, MD; \\ Peter H. Hackett, MD \\ From the IFREMMONT: Institut de Recherche et de Formation en Medecine de Montagne, Hôpital de Chamonix, Chamonix, France (Dr Cauchy); \\ the Hôpitaux des Pays du Mont-Blanc, Sallanches, France (Dr Cauchy); the University of Colorado Denver School of Medicine, Department \\ of Emergency Medicine, Aurora, CO (Drs Davis and Hackett); the Emergency Service, Lausanne University Hospital, Lausanne, Switzerland \\ (Dr Pasquier); the Air-Glaciers SA and GRIMM, Sion, Switzerland (Dr Pasquier); the Rural Partners in Medicine, LLC, Steamboat Springs, \\ (Dr Meyer); and the Institute for Altitude Medicine, Telluride, CO (Dr Hackett).
}

\begin{abstract}
Despite advances in outdoor clothing and medical management of frostbite, individuals still experience catastrophic amputations. This is a particular risk for those in austere environments, due to resource limitations and delayed definitive treatment. The emerging best therapies for severe frostbite are thrombolytics and iloprost. However, they must be started within 24 hours after rewarming for recombinant tissue plasminogen activator (rt-PA) and within 48 hours for iloprost. Evacuation of individuals experiencing frostbite from remote environments within 24 to 48 hours is often impossible. To date, use of these agents has been confined to hospitals, thus depriving most individuals in the austere environment of the best treatment. We propose that thrombolytics and iloprost be considered for field treatment to maximize chances for recovery and reduce amputations. Given the small but potentially serious risk of complications, rt-PA should only be used for grade 4 frostbite where amputation is inevitable, and within 24 hours of rewarming. Prostacyclin has less risk and can be used for grades 2 to 4 frostbite within 48 hours of rewarming. Until more field experience is reported with these agents, their use should probably be restricted to experienced physicians. Other modalities, such as local nerve blocks and improving oxygenation at high altitude may also be considered. We submit that it remains possible to improve frostbite outcomes despite delayed evacuation using resource-limited treatment strategies. We present 2 cases of frostbite treated with rt-PA at K2 basecamp to illustrate feasibility and important considerations.
\end{abstract}

Key words: frostbite, prostacyclin, thrombolytics, field treatment

\section{Introduction}

Even with today's advances in improved outdoor clothing, preventive efforts, and medical management, severe frostbite still occurs, often resulting in catastrophic amputations. The risk of amputation is particularly high when injuries occur in the austere environment, where resources are limited. Groups at increased risk include winter recreationists such as alpinists, skiers, snowmobilers, and polar travelers, as well as those working or assigned to cold-weather operations, including the military. The main reason for poor outcome in this environment is usually delay in definitive care, which is

Presented at the 19th International Hypoxia Symposium, March 4, 2015, Lake Louise, Canada.

Corresponding author: Emmanuel Cauchy, MD, IFREMMONT: Institut de Recherche et de Formation en Medecine de Montagne, Hôpital de Chamonix, 509 route des Pélerins 74400 Chamonix France (e-mail: cauchy@ifremmont.com). often unavoidable. The emerging use of thrombolytics and iloprost offers the first major advances in the treatment of frostbite in decades and has reduced the rate of amputation. However, these therapies have been confined to hospital use. Given the remote locations in which many of these injuries occur, and the urgency of timely treatment, serious consideration should be given to employing these treatments in the prehospital setting under certain conditions. In this article, we discuss the field management of severe frostbite, and the indications, risks, and methods for use of thrombolytics or prostacyclin by a trained physician. We also suggest the possible adjunctive therapies of oxygenation at high altitude, and peripheral nerve block.

\section{Field Management of Frostbite}

Field treatment of frostbite remains challenging for several reasons. First is the difficulty of assessing the 
severity of injury, a crucial determination that dictates management and evacuation decisions. Second is determining the time of onset of injury, duration of freezing, and time since thawing in those who have spontaneously thawed. Other difficulties include the logistics of evacuation to an appropriate facility, and the presence of comorbid conditions such as hypothermia, trauma, or medical illness. In addition, treatment greatly depends on medical capabilities and available supplies, a factor that has not previously been adequately considered in treatment algorithms for field management of frostbite. Finally, the most promising therapies for severe frostbite have been confined to hospital use and are unavailable, impractical, or inappropriate for field use. Tragically, too many patients reach the hospital too late for successful use of medical therapy.

\section{EVALUATION OF THE SEVERITY OF THE INJURY AND AMPUTATION RISK}

In both the field and hospital, evaluation of frostbite injury is based on presence or absence of perfusion after rewarming, combined with extent of nonperfused tissue. For assessing perfusion, careful examination includes skin color and temperature, sensation, pulses, and capillary refill. Expedition basecamps or field clinics as well as hospitals may have fast-response infrared thermometers, pulse oximeters, and even Doppler devices. The newer high-frequency Doppler devices offer marked improvement for assessing perfusion, and are used to assess microcirculation intraoperatively and in various grafts and flaps. ${ }^{1,2}$ Handheld battery-powered models are available. Level of nonperfusion or discoloration can be described with the usual hand/foot anatomy of joints: metacarpophalangeal, proximal interphalangeal, distal interphalangeal, or tuft. A number of classification systems may be used to evaluate severity. ${ }^{3,4}$ We offer a practical classification of frostbite injuries in the Figure, with details in a previous publication. ${ }^{5}$ Although bone amputation risk depends on lack of perfusion to the bone, which cannot be determined in the field, soft tissue ischemia and cyanosis are surrogate markers for amputation risk, although not at the same level. That is, the eventual level of bone amputation is always distal to the boundary between normal color and cyanotic discoloration of the digit. After rewarming, a digit with cyanosis that extends proximal to the proximal interphalangeal joint must be considered at high risk for amputation distal to that joint; the greater the extent of cyanosis, the greater the risk of amputation (Figure). At high altitude, the risk of poor outcome may increase due to hypoxemia $\left(\mathrm{Spo}_{2}<90 \%\right.$, c. $\left.4000 \mathrm{~m}\right)$, less vasodilation in the cold, dehydration, etc.; thus, grade 2

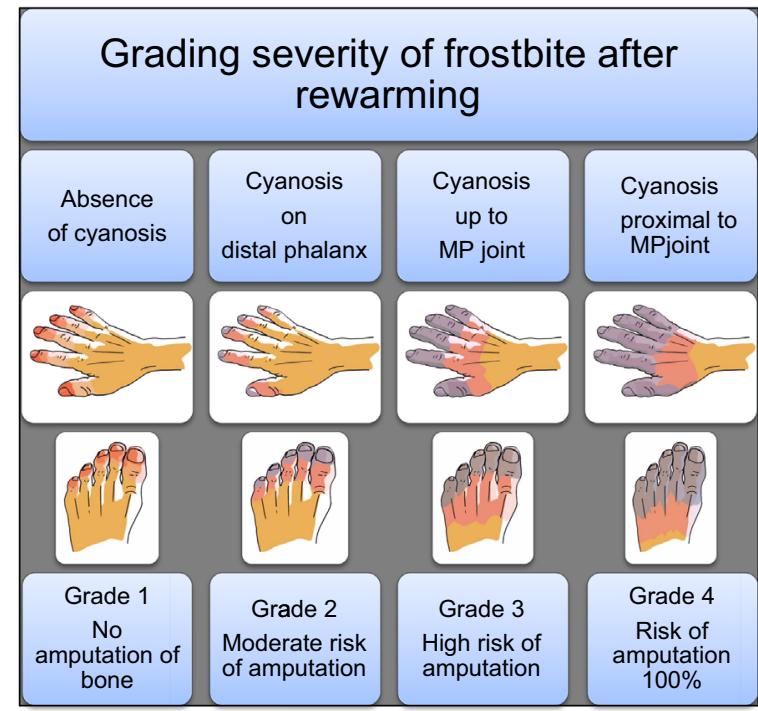

Figure. Grading severity of frostbite and bone amputation risk after rewarming.

injuries, based on our experience, should be considered at higher risk for amputation at high altitude compared with low altitude. In the hospital setting, grading of frostbite severity is easier than in the field, due to the comfort and safety of the environment, availability of easy rapid rewarming, and observation over time. Although some practitioners obtain nuclear bone scans on day 0 , the literature suggests that scanning only on day 2 or later improves prognostication. ${ }^{6}$

\section{BASIC FIELD CARE}

A recent publication of frostbite management guidelines includes several important treatments for use in the field. $^{7}$ For example, the individual should rehydrate orally with warm fluids, and the injured extremity should be rewarmed by immersion in a 37 to $39^{\circ} \mathrm{C}$ water bath if there is low risk of refreezing. In order to treat hyperviscosity and inflammation, the guidelines recommend $12 \mathrm{mg} / \mathrm{kg} / \mathrm{day}$ of ibuprofen, and others recommend aspirin. ${ }^{8}$ In addition, low molecular weight dextran is a grade $2 \mathrm{C}$ recommendation for use when thrombolytics or iloprost are not going to be used. However, low molecular weight dextran is of questionable value and can cause anaphylaxis; we do not recommend it. If blisters appear, a dressing is suggested. Systemic antibiotics should be considered for severe frostbite (grades 3-4) in special circumstances. Others have suggested that for foot frostbite, anticoagulants should be considered to avoid the complications of thrombosis and phlebitis, especially if the individual is nonambulatory. ${ }^{9}$ 
Recently, 2 new ideas for field treatment have been suggested but not yet studied: prehospital distal sympathetic nerve block, and increased oxygenation for frostbitten patients who are at high altitude, or hypoxic for any reason. ${ }^{10,11}$

\section{Locoregional anesthesia (peripheral nerve blocks)}

Extensive literature, primarily in hand surgery, confirms that upper extremity sympathetic nerve blocks cause vasodilation and increased skin temperature of the fingers. ${ }^{12}$ Whether this would produce similar results in frostbitten hands or fingers is unknown but has been suggested. ${ }^{13-15}$ Cervical, axillary, and brachial nerve blocks and intra-arterial (IA) vasodilators have been used for frostbite treatment previously, but without controlled trials, and only in hospitals. ${ }^{16,17}$ The results have been mixed, and the Wilderness Medical Society guidelines do not recommend sympathectomy because of insufficient evidence. However, it is possible that very early intervention with sympathetic blockade in the field may be more effective. Pasquier et al recently described a simple technique that was used for field care of grade 2 bilateral hand frostbite in an alpinist. Bilateral nerve blocks were performed just proximal to the wrist with ropivacaine. ${ }^{10}$ Pain in both hands was completely relieved in 10 minutes and recovery was excellent, without amputation. This technique deserves proper study and may be considered in the field treatment of grades 2 to 4 frostbite, for both pain control and vasodilation, by those competent in the procedure.

\section{Oxygenation or portable recompression chamber}

Whether increasing arterial oxygenation in hypoxic persons at high altitude $\left(\mathrm{Spo}_{2}<90 \%\right.$, usually $\geq 4000$ $\mathrm{m})$ is helpful for frostbite injuries is unknown. Although it seems reasonable to assume that improved arterial oxygen content may help, the lack of perfusion to frostbitten tissue may render this therapy futile. However, if oxygen was to promote vasodilatation and improve perfusion, it would be valuable. In contrast, if oxygen therapy was to cause vasoconstriction, as it does at low altitude, it would be counterproductive. Coldinduced vasodilation is considered a protective mechanism for cold injuries, and usually the changes in peripheral blood flow during cold exposure are in accordance with the cold-induced vasodilation response. ${ }^{18}$ Acute hypoxia tends to diminish coldinduced vasodilation, with marked impairment of hand rewarming, while acclimatization of more than 1 week tends to augment the response. ${ }^{19,20}$ Thus the effect of supplemental oxygen or hyperbaric therapy on peripheral blood flow may depend on the duration of hypoxic exposure. Until we know that oxygen therapy will not cause peripheral vasoconstriction, we should perhaps be cautious. However, portable recompression chambers and supplemental oxygen only provide a reduction of hypoxemia and not hyperoxia. A final consideration is whether at extremely high altitude, supplemental oxygen may help increase basal metabolic rate and also produce peripheral vasodilatation. The sensation of severe chill replaced by warmth during oxygen breathing when over $8000 \mathrm{~m}$ is so commonly reported by mountaineers that it must be considered. This could be multifactorial. For example, oxygen allows the climber to increase oxygen consumption, generate more body heat, increase core temperature, and thus reduce the stimulus for peripheral vasoconstriction. Any measure to increase core temperature or prevent its drop will help prevent frostbite and perhaps contribute to its treatment as well. We feel that at extreme altitude, oxygenation may be important in the approach to frostbite, as well as important for prevention, although at more moderate altitude $(4000-6000 \mathrm{~m})$, its potential benefit is less clear and needs to be evaluated. The arterial partial pressure of oxygen can be increased in the field by either breathing supplemental oxygen or by use of a portable hyperbaric chamber. Whether these modalities may be different in terms of influencing peripheral blood flow is also unknown and is an issue worthy of investigation.

\section{Thrombolytic and vasodilator pharmacological treatment}

In the hospital, after rehydration, rewarming, and addressing tetanus status, several different therapeutic approaches may be considered. Thrombolytic protocols using streptokinase, urokinase, or more recently recombinant tissue plasminogen activator (rt-PA) by intravenous (IV) or IA route have been successfully implemented and published, although not yet validated by randomized controlled trials. ${ }^{21-26}$ Although IA rt-PA requires invasive angiography and is thus limited to certain facilities, thrombolysis with intravenous rt-PA is already used in prehospital care in many countries for myocardial infarction. The incidence of serious bleeding complications when used for acute myocardial infarction is less than $1 \%{ }^{27}$ When given in cases of stroke mimics, the symptomatic intracranial hemorrhage rate is about $1 \%$. There is no evidence that IV administration has greater risk compared with IA in treatment of peripheral arterial occlusion. ${ }^{28}$ Limited data suggest that to be most efficacious in frostbite, rt-PA should be administered within 6 to 24 hours of rewarming. ${ }^{21,22,24,29,30}$

Iloprost was used in 6 subjects in 2 case reports, and a recent randomized controlled trial with 47 subjects found 
that IV iloprost is effective in reducing amputations up to 48 hours after rewarming and has a much better safety profile than rt-PA. ${ }^{31-33}$ The patient receiving iloprost must be supine to avoid postural hypotension. Side effects include flushing, headaches, nausea, and occasionally vomiting; therefore, the fasting state is preferred. Serious adverse reactions are uncommon; in 81 treatments in Chamonix, infusion had to be stopped only 3 times, once for palpitations and twice for vomiting. To date, iloprost has not been used out of the hospital. Medical or surgical sympathectomy has yielded mixed results and seems to be more useful in managing longterm sequelae. ${ }^{34,35}$ Hyperbaric oxygen therapy has been used sporadically, has not yet been studied in controlled trials, and is not recommended in current guidelines for lack of sufficient data. ${ }^{7}$

A key issue is proper patient selection for thrombolytics or prostacyclin treatment. Duration of injury and thawing may be critical. Although some authors have suggested that digits frozen for more than 24 hours precludes aggressive therapy, ${ }^{27}$ we recommend using time from thawing rather than freezing duration for management decisions; we are in agreement with recent guidelines and other experts. ${ }^{5,22}$ In reality, the duration of freezing is often very difficult to determine. Patients may recall when their digits became numb, for example, but time of actual freezing is usually unclear. Given the terrible impact of amputations, and the chance of better results with aggressive treatment, we do not recommend duration of freezing beyond which treatment would be considered absolutely unnecessary or futile. The emerging data on use of thrombolytics suggest that there is little value when used more than 24 hours after thawing, while prostacyclin was useful up to 48 hours after thawing. ${ }^{5,31}$ Other factors in patient selection have to do with comorbidities and contraindications to thrombolytics or prostacyclin use (Tables 1 and 2).

Transitioning new therapies from the hospital to the field also must take into account the available resources and skills of the provider. These therapies require monitoring capabilities, familiarity with the medications and their possible complications, and the use of some specialized equipment, such as a syringe pump. They are thus more suited for large expedition basecamps and field clinics with medical expertise, rather than small groups with more limited resources. Although it would be impractical for rescue groups to start prostacyclin or rt-PA during rescue or evacuation, it may be possible for a well-equipped and staffed team that has reached a patient but then is faced with delayed evacuation.

Considering that rapid evacuation to hospitals remains impractical in many remote areas, we propose here a
Table 1. Protocol for intravenous rt-PA

\begin{tabular}{lc}
\hline Administration & Weight $<67 \mathrm{~kg}: 15 \mathrm{mg}$ IV \\
& bolus, then $0.75 \mathrm{mg} / \mathrm{kg}$ over \\
& 30 minutes, then $0.35 \mathrm{mg} / \mathrm{kg}$ \\
over next $60 \mathrm{minutes}$ & Weight $>67 \mathrm{~kg}: 15 \mathrm{mg} \mathrm{IV}$ \\
Ideally given & bolus, then $50 \mathrm{mg}$ over 30 \\
with a portable & minutes, then $35 \mathrm{mg}$ over \\
syringe pump & next 60 minutes. Total not to \\
& exceed $100 \mathrm{mg}$ \\
Heparin after bolus & Recent trauma, bleeding \\
diathesis, stroke \\
within 3 months, on \\
anticoagulants, \\
hypersensitivity; BP $>180$ \\
mmHg systolic or \\
110 mmHg diastolic \\
High altitude: HAPE or HACE, \\
retinal hemorrhage, gastritis \\
Bleeding: stop infusion, \\
hemostasis if possible, \\
consider tranexamic acid \\
Angioedema: stop infusion, \\
antihistamine, corticosteroids \\
Complications and
\end{tabular}

rt-PA, recombinant tissue plasminogen activator; IV, intravenous; $\mathrm{BP}$, blood pressure; HAPE: high altitude pulmonary edema; HACE: high altitude cerebral edema.

novel field treatment pathway that incorporates the recent advances in frostbite treatment. This pathway is dichotomized based on the medical training of the care providers.

\section{PRACTICAL APPROACH IN THE FIELD}

\section{Treatment by persons with limited medical background}

Basic care. All the traditional actions to correct or maintain core temperature and stabilize the individual should be taken, including obtaining shelter from the elements. Wet clothes and boots should be removed when practical. The individual should be given warm fluids and dry clothing or a sleeping bag if available. Rubbing the affected part is not recommended because of the potential for worsening direct tissue injury. See recent guidelines for details of basic care. ${ }^{7}$

Warm water immersion. If the affected tissue is still frozen or partly frozen, if refreezing is not a risk, and the equipment is available, the affected extremities should be immersed in a 37 to $39^{\circ} \mathrm{C}$ water bath as soon as possible. Immersion should last 30 to 60 minutes or until 
Table 2. Protocol for intravenous prostacyclin

\begin{tabular}{ll}
\hline Administration and monitoring & Dilute 1 vial $0.5 \mathrm{~mL}$ iloprost in $24.5 \mathrm{~mL} \mathrm{NaCl} 9 \%$ \\
& Syringe pump: $25 \mathrm{~mL}-$ speed: $1 \mathrm{~mL} / \mathrm{h}$ for 30 minutes, then $2 \mathrm{~mL} / \mathrm{h}$ for $30 \mathrm{minutes}$, \\
& then $3 \mathrm{~mL} / \mathrm{h}$ for 30 minutes, then $4 \mathrm{~mL} / \mathrm{h}$ for weight $<75 \mathrm{~kg}$ or $5 \mathrm{~mL} / \mathrm{h}$ for weight \\
& $>75 \mathrm{~kg}$ \\
& Continue until $25 \mathrm{~mL}$ is delivered; all patients receive 1 vial \\
& Monitor $\mathrm{HR}$ and $\mathrm{BP}$ every 30 minutes \\
Complications and their management & In case of side effects decrease to previous lower step \\
& If systolic $\mathrm{BP}<90 \mathrm{mmHg}$ decrease to lower step \\
Contraindications & Hypotension, hypersensitivity, pulmonary edema, cardiac arrhythmia, \\
Precautions & active ulcer disease, major trauma; unknown effects on pregnancy \\
& Anticipate nausea and vomiting, pain and hypotension; keep patient supine
\end{tabular}

$\mathrm{HR}$, heart rate; $\mathrm{BP}$, blood pressure.

a red/purple color appears on the skin, and distal flushing is maximal.

Other rewarming methods. If rapid rewarming in a water bath is not possible, digits can be rewarmed by placing them on another's warm skin, such as in the axilla or groin, or with the use of hand warmers or heat packs. Although these devices are used commonly for prevention of cold injuries and to improve comfort of hands and feet, their use in frostbite has not been evaluated, and caution is warranted because of lack of temperature control and possible skin trauma. Other heat sources such as fire, electrical heaters, and ovens are discouraged because of risk of thermal injury. ${ }^{7}$ There are often delays in diagnosis of frostbite and by the time of diagnosis, spontaneous thawing has often occurred. In such cases, rapid warming may not be as important, but it offers a chance to maximize vasodilatation and to clean the injured area; therefore, we recommend it.

Evaluation. After initial assessment and basic care, and after rapid rewarming if that was employed, we suggest grading the severity of frostbite according to the Figure.

Medication. If not contraindicated (allergy, active ulcer disease), 75 to $81 \mathrm{mg}$ of aspirin or 400 to $600 \mathrm{mg}$ of ibuprofen 2 to 3 times a day (or $12 \mathrm{mg} / \mathrm{kg} /$ day) should be given for its antiprostaglandin and analgesic effect. ${ }^{30}$ Analgesics may be required on an individual basis (paracetamol, tramadol, opiates).

Oxygen or hyperbaric therapy. At high altitude when arterial oxygen saturation is less than $90 \%$ (usually $\geq 4000$ meters) oxygen or a hyperbaric device might be considered if available. ${ }^{36}$ Supplemental oxygen can be titrated to maintain $\mathrm{Spo}_{2}$ to $90 \%$ or greater, and a comparable increase in oxygenation can be accomplished with hyperbaria, depending on the altitude. Both treatments would seem most important during the rewarming protocol, although rewarming in a hyperbaric chamber is impractical. One suggestion is that just after rapid rewarming, 1-hour sessions in a portable hyperbaric chamber can be repeated every 3 hours during the first 12 hours while awaiting evacuation. ${ }^{11}$ Descent also accomplishes the goal of increasing arterial oxygenation.

\section{TREATMENT BY PERSONS WITH MEDICAL CAPABILITIES}

In addition to the basic treatment recommended for nonmedical personnel, several other medications can be considered. Although not proven effective for frostbite, calcium channel blockers and PDE-5 inhibitors are vasodilators that provide significant benefit in Raynaud's phenomena and are often available in field medical kits, especially at high altitude. ${ }^{37-40}$ Authors recommended various regimens. A reasonable dosing schedule is sildenafil 50 to $100 \mathrm{mg}$ twice a day, tadalafil $10 \mathrm{mg}$ twice a day, or nifedipine $30 \mathrm{mg}$ slow release twice a day; these medications should not be combined. Some patients may also require narcotic analgesics during or after rewarming. An injectable nonsteroidal antiinflammatory drug, such as ketorolac, may provide quicker onset of action than oral ibuprofen, avoid nausea and vomiting, and may be especially useful if the individual's digits are still frozen upon presentation, because nonsteroidal anti-inflammatory drugs have been shown in animal models to preserve frostbitten tissue if given before thawing.

$r t-P A$

Physicians can consider using intravenous rt-PA in the field when dealing with frostbite that will clearly result in amputation if untreated (grade 4), especially in the hands, and with multiple digits. The dose and considerations for its use are in Table 1. 
Should any suspicion of severe trauma, intracranial or clinically significant hemorrhage occur in the field, the infusion should be discontinued. Managing a hemorrhagic thrombolysis-associated complication in the field would be challenging, as it is even in an in-hospital setting. Antifibrinolytic agents may be considered in this situation (eg, tranexamic acid $1 \mathrm{~g}$ ). Angioedema, an occasional complication of rt-PA thrombolysis $(1.3 \%-5 \%)$, should be treated with antihistamine and corticosteroid. ${ }^{41}$

\section{Iloprost infusion}

Iloprost is a synthetic analogue of prostacyclin PGI2. It dilates systemic and pulmonary arterial vascular beds and also affects platelet aggregation

Iloprost is most commonly used for pulmonary arterial hypertension, scleroderma, and Raynaud's phenomenon. It is available in inhaled and IV forms, but is not available in the IV form in many countries, including the United States. Whether the inhaled form may be useful for the peripheral circulation is unknown and requires investigation. Only the IV form has been used for frostbite to date. Because iloprost entails less risk than rt-PA, it should be considered for grades 2 to 4 frostbite, especially in the hands. In such cases, a trained medical practitioner with emergency skills can initiate field iloprost treatment if blood pressure monitoring and a portable syringe pump are available. Such equipment should be made available in expedition basecamps, field clinics for the military or commercial operations, and local health care clinics in remote areas where evacuation is likely to be delayed. In our study over 22 years there were no severe side effects, although a transitory drop of blood pressure, headaches, and vomiting are possible. ${ }^{33}$ See Table 2 for details of administration. When diluted to $25 \mathrm{~mL}$ solution, an hourly rate of infusion is used (Table 2). An alternative is to start with $0.5 \mathrm{ng} / \mathrm{kg} / \mathrm{min}$ and increase every 30 minutes by $0.5 \mathrm{ng} /$ $\mathrm{kg} / \mathrm{min}$ up to $2 \mathrm{ng} / \mathrm{kg} / \mathrm{min}$. If the patient develops unacceptable side effects, the rate is then reduced by $0.5 \mathrm{ng} / \mathrm{kg} / \mathrm{min}$. The infusion with either protocol is continued until the contents of 1 vial are consumed (50 mcg iloprost per vial). That is, all patients received a total of $50 \mathrm{mcg}$ iloprost, regardless of weight, usually over 5 to 6 hours; the exact time depends on tolerance of infusion rate. The patient should be evacuated after this 1 dose. However, if the patient remains in the field, ideally the dose should be repeated each day for a total of 3 days Because the first dose seems to be the most important, it should be given even if further doses are not possible. In contrast to thrombolytics, iloprost can be used when there is a history of moderate trauma or when the thawing occurred over 24 hours ago (in our experience, up to 48 hours postthaw). Analgesic medication for headaches and antiemetics is sometimes necessary.

We present 2 case histories of climbers treated for frostbite at K2 basecamp by one of the authors (EFM).

\section{Patient 1}

A 42-year-old man made an open bivouac at 7400 meters after summiting K2. During the night, he awoke to find there was no glove on his left hand. He descended to base camp wearing a glove and was evaluated approximately 14 hours after the start of his bivouac. Exact time of freezing and thawing was unknown; the hand had spontaneously thawed by the time he reached basecamp. Examination revealed grade 3 frostbite with purplish discoloration of the fingers of the left hand, to between the metacarpophalangeal and proximal interphalangeal joints. All the fingers were moderately swollen. No sensation and no capillary refill were detected in any of the digits. Although it was already thawed, the physician warmed the hand in water at $39^{\circ} \mathrm{C}$ for 3 hours. Between 30 and 60 minutes of warming, the physician established an IV and administered $5 \mathrm{mg}$ IV morphine sulfate, $5 \mathrm{mg}$ IV diazepam (for agitation), and $800 \mathrm{mg}$ ibuprofen orally. At 90 minutes of warming, he administered intravenous rt-PA, $1.4 \mathrm{mg} / \mathrm{kg}$ over 15 minutes, followed by intravenous heparin 1000 units per hour for 4 hours. No topical medications were applied. There were no immediate or late complications, and recovery was excellent. At follow-up in Europe, he had only sloughing of superficial skin.

\section{Patient 2}

A 40-year-old male climber sustained frostbite of both feet after reaching the summit of $\mathrm{K} 2$. He presented at basecamp 2.5 days later, 60 to 66 hours after freezing; the feet had thawed during descent, exact timing unknown. Examination showed grade 4 frostbite, with discoloration and swelling of all toes into the midmetatarsals of both feet, with no capillary refill or sensation to the ankles. He was treated exactly the same as Patient 1. Several days later, he returned to Europe and all toes were eventually amputated. No complications of thrombolytic therapy were observed either immediately or late.

\section{Comment}

Thrombolytic therapy was utilized safely at K2 basecamp. Although the dosage of rt-PA was slightly different from current recommendations, these cases demonstrate that its use is feasible in this extreme environment. Although only 2 patients were treated, it is reassuring that there were no complications. The 
therapy likely prevented amputations for Patient 1 , while Patient 2 was probably outside the therapeutic window of 24 hours after thawing and there was no benefit of thrombolytic therapy. These case examples should help encourage the use of thrombolytics therapy in the field, when the risk of amputation is high, no contraindications are present, and the physician is familiar with the therapy. We hope there will soon be a report of frostbite injury in the field with prostacyclin.

\section{Conclusion}

In cases of severe frostbite with delayed evacuation, the choice of treatment depends on 3 parameters: the severity of frostbite, the resources available, and the medical experience of the provider. Basic care including rewarming and ibuprofen or aspirin administration can be dispensed in the field. Oxygen or portable recompression chamber may be useful at very high altitudes by any rescuer. Other management options for experienced or trained physicians include thrombolytics or prostacyclin infusion, and locoregional anesthesia. Because of the risk of complications, rt-PA should only be used for severe and extended frostbite (grade 4) rewarmed within 24 hours, while prostacyclin, being safer, can be used for up to 48 hours postthawing in grades 2 to 4 frostbite. Introduction of these new therapies for field use will likely result in fewer amputations, and studies assessing these treatments will hopefully be forthcoming.

\section{References}

1. Kaspar M, Partovi S, Aschwanden M, et al. Assessment of microcirculation by contrast-enhanced ultrasound: a new approach in vascular medicine. Swiss Med Wkly. 2015; 145:w14047.

2. Toprak U, Hayretci M, Erhuner Z, et al. Dynamic Doppler evaluation of the hand arteries to distinguish between primary and secondary raynaud phenomenon. AJR Am J Roentgenol. 2011;197:W175-180.

3. Foray J. Frostbite [in French]. Ann Dermatol Venereol. 2001;128:1075-1080.

4. Mills WJ Jr. Summary of treatment of the cold injured patient: frostbite. 1983. Alaska Med. 1993;35:61-66.

5. Cauchy E, Chetaille E, Marchand V, Marsigny B. Retrospective study of 70 cases of severe frostbite lesions: a proposed new classification scheme. Wilderness Environ Med. 2001;12:248-255.

6. Cauchy E, Chetaille E, Lefevre M, Kerelou E, Marsigny B. The role of bone scanning in severe frostbite of the extremities: a retrospective study of 88 cases. Eur $J$ Nucl Med. 2000;27:497-502.

7. McIntosh SE, Hamonko M, Freer L, et al. Wilderness Medical Society practice guidelines for the prevention and treatment of frostbite. Wilderness Environ Med. 2011;22: $156-166$.
8. Zafren K. Frostbite: prevention and initial management. High Alt Med Biol. 2013;14:9-12.

9. Foray J, Foray P, Abrassart S. Mountain frostbite (apropos of 1260 recorded cases) [in French]. Schweiz Z Sportmed. 1991;39:6-11.

10. Pasquier M, Ruffinen GZ, Brugger H, Paal P. Pre-hospital wrist block for digital frostbite injuries. High Alt Med Biol. 2012;13:65-66.

11. Cauchy E, Leal S, Magnan MA, Nespoulet H. Portable hyperbaric chamber and management of hypothermia and frostbite: an evident utilization. High Alt Med Biol. 2014;15:95-96.

12. Hermanns H, Braun S, Werdehausen R, Werner A, Lipfert $\mathrm{P}$, Stevens MF. Skin temperature after interscalene brachial plexus blockade. Reg Anesth Pain Med. 2007;32:481-487.

13. Calder K, Chung B, O'Brien C, Lalonde DH. Bupivacaine digital blocks: how long is the pain relief and temperature elevation? Plast Reconstr Surg. 2013;131:1098-1104.

14. Chandran GJ, Chung B, Lalonde J, Lalonde DH. The hyperthermic effect of a distal volar forearm nerve block: a possible treatment of acute digital frostbite injuries? Plast Reconstr Surg. 2010;126:946-950.

15. Shapovalov KG, Burdinskii EN, Stepanov AV. Optimization of the components of regulation of vascular tone and microcirculatory hemostasis during prolonged regional block in local cold injury [in Russian]. Anesteziol Reanimatol. 2008;3:20-22.

16. Koster U, Mutschler B, Hempel V. Treatment of frostbite of the extremities by combined axillary plexus and peridural anesthesia: a case report including blood levels of bupivacaine [in German]. Reg Anaesth. 1987;10:93-95.

17. Syposs T, Novak J, Nagy L, Keresztes L. Management of frostbite of the lower limbs by continuous epidural blockade [in Hungarian]. Orv Hetil. 1986;127:27-30.

18. Mathew L, Purkayastha SS, Selvamurthy W, Malhotra MS. Cold-induced vasodilatation and peripheral blood flow under local cold stress in man at altitude. Aviat Space Environ Med. 1977;48:497-500.

19. Daanen HA, van Ruiten HJ. Cold-induced peripheral vasodilation at high altitudes - a field study. High Alt Med Biol. 2000;1:323-329.

20. Keramidas ME, Kolegard R, Mekjavic IB, Eiken O. Acute effects of normobaric hypoxia on hand-temperature responses during and after local cold stress. High Alt Med Biol. 2014;15:183-191.

21. Twomey JA, Peltier GL, Zera RT. An open-label study to evaluate the safety and efficacy of tissue plasminogen activator in treatment of severe frostbite. J Trauma. 2005:59:1350-1354. discussion 4-5.

22. Bruen KJ, Ballard JR, Morris SE, Cochran A, Edelman LS, Saffle JR. Reduction of the incidence of amputation in frostbite injury with thrombolytic therapy. Arch Surg. 2007;142:546-551. discussion 51-53.

23. Johnson AR, Jensen HL, Peltier G, DelaCruz E. Efficacy of intravenous tissue plasminogen activator in frostbite patients and presentation of a treatment protocol for frostbite patients. Foot Ankle Spec. 2011;4:344-348. 
24. Mohr WJ, Jenabzadeh K, Ahrenholz DH. Cold injury. Hand Clin. 2009;25:481-496.

25. Gonzaga T, Jenabzadeh K, Anderson CP, Mohr WJ, Endorf FW, Ahrenholz DH. Use of intraarterial thrombolytic therapy for acute treatment of frostbite in 62 patients with review of thrombolytic therapy in frostbite. $J$ Burn Care Res. 2015 Apr 24. http://dx.doi.org/10.1097/BCR. 0000000000000245 . Accessed May 5, 2015.

26. Ibrahim AE, Goverman J, Sarhane KA, Donofrio J, Walker TG, Fagan SP. The emerging role of tissue plasminogen activator in the management of severe frostbite. J Burn Care Res. 2015;36:e62-e66.

27. American College of Emergency Physicians, Society for Cardiovascular Angiography and Interventions, 2013 ACCF/AHA guideline for the management of STelevation myocardial infarction: a report of the American College of Cardiology Foundation/American Heart Association Task Force on Practice Guidelines. J Am Coll Cardiol. 2013;61:e78-e140.

28. Robertson I, Kessel DO, Berridge DC. Fibrinolytic agents for peripheral arterial occlusion. Cochrane Database Syst Rev. 2010(3):. CD001099.

29. Bruen KJ, Gowski WF. Treatment of digital frostbite: current concepts. J Hand Surg Am. 2009;34:553-554.

30. Handford C, Buxton P, Russell K, et al. Frostbite: a practical approach to hospital management. Extrem Physiol Med. 2014;3:7.

31. Hödl S. Treatment of freezing injury [in German]. Wien Med Wochenschr. 2005;155:199-203.

32. Groechenig E. Treatment of frostbite with iloprost. Lancet. 1994;344:1152-1153.
33. Cauchy E, Cheguillaume B, Chetaille E. A controlled trial of a prostacyclin and rt-PA in the treatment of severe frostbite. N Engl J Med. 2011;364:189-190.

34. Bouwman DL, Morrison S, Lucas CE, Ledgerwood AM. Early sympathetic blockade for frostbite-is it of value? J Trauma. 1980;20:744-749.

35. Taylor MS. Lumbar epidural sympathectomy for frostbite injuries of the feet. Mil Med. 1999;164:566-567.

36. Imray C, Grieve A, Dhillon S, Caudwell Xtreme Everest Research Group, Cold damage to the extremities: frostbite and non-freezing cold injuries. Postgrad Med J. 2009;85: 481-488.

37. Distler JH. Primary and secondary Raynaud's phenomenon [in German]. Z Rheumatol. 2008;67:211-217. quiz 8-9.

38. Rosenkranz S, Diet F, Karasch T, Weihrauch J, Wassermann K, Erdmann E. Sildenafil improved pulmonary hypertension and peripheral blood flow in a patient with scleroderma-associated lung fibrosis and the raynaud phenomenon. Ann Intern Med. 2003;139:871-873.

39. Roustit M, Blaise S, Allanore Y, Carpentier PH, Caglayan E, Cracowski JL. Phosphodiesterase-5 inhibitors for the treatment of secondary Raynaud's phenomenon: systematic review and meta-analysis of randomised trials. Ann Rheum Dis. 2013;72:1696-1699.

40. Roustit M, Hellmann M, Cracowski C, Blaise S, Cracowski JL. Sildenafil increases digital skin blood flow during all phases of local cooling in primary Raynaud's phenomenon. Clin Pharmacol Ther. 2012;91:813-819.

41. Fugate JE, Rabinstein AA. Update on intravenous recombinant tissue plasminogen activator for acute ischemic stroke. Mayo Clin Proc. 2014;89:960-972. 